\title{
A Model for the Quantization of the Hall Resistance in the Quantum Hall Effect
}

\author{
Paul Bracken \\ Department of Mathematics, University of Texas, Edinburg, USA \\ E-mail: bracken@panam.edu \\ Received January 25, 2010; revised February 26, 2010; accepted March 20, 2010
}

\begin{abstract}
Some aspects of anyon physics are reviewed with the intention of establishing a model for the quantization of the Hall conductance. A single particle Schrödinger model is introduced and coupled with a constraint equation formulated from the anyon picture. The Schrödinger equation-constraint system can be converted to a single nonlinear differential equation and solutions for the model can be produced.
\end{abstract}

Keywords: Anyon, Hall Resistance, Conductance, Composite Particles

\section{Introduction}

The quantum Hall effect is a recently discovered and now well known phenomenon which appears in a twodimensional electron system which exhibits spectacular phenomena when subjected to an intense transverse magnetic field [1]. First encountered experimentally [2], the integer quantum Hall effect has received much study, and was subsequently followed by the fractional Hall effect $[3,4]$. Referring to these two effects as the quantum Hall effect, the Hall resistance is found experimentally to exhibit plateaus at the quantized values

$$
R_{H}=\frac{h}{f e^{2}}
$$

where $f$ is either an integer or a simple rational fraction. Thus (1) incorporates both of these effects. For the integer case, $f$ takes on integer values $f=n=$ $1,2,3, \cdots$, and some prominent fractions for the fractional case appear in sequences such as $f=\frac{n}{2 n \pm 1}, \frac{n}{4 n \pm 1}$. The two effects show remarkable similarities despite the differences in origin. In both effects, the localization of electrons and quasiparticles is believed to be responsible for the formation of the plateaus in the Hall conductivity. At the transitions between successive plateaus in the integer quantum Hall effect, scaling behavior has been observed. Theoretically, the aim in understanding this is to solve the many-body quantum mechanical problem defined by the many body Hamiltonian given by

$$
H=\sum_{\mathrm{j}} \frac{1}{2 \mathrm{~m}_{\mathrm{e}}}\left[\overrightarrow{\mathrm{p}_{\mathrm{j}}}+\frac{\mathrm{e}}{\mathrm{c}} \overrightarrow{\mathrm{A}}\left(\overrightarrow{\mathrm{r}_{\mathrm{j}}}\right)\right]^{2}+\frac{1}{2} \sum_{\mathrm{j} \neq \mathrm{k}} \frac{\mathrm{e}^{2}}{\epsilon \mathrm{r}_{\mathrm{jk}}}+\sum_{\mathrm{j}} \mathrm{U}\left(\overrightarrow{\mathrm{r}_{\mathrm{j}}}\right)
$$

The first term on the right-hand side is the kinetic energy in the presence of a constant external magnetic field; the second term is the Coulomb interaction energy; and the third term is a one-body potential due to a uniform positive background. The electrons are constrained to move in the two-dimensional $x y$-plane.

It is the intention here to set up and solve a simple version of (2) subject to a physical constraint. Such pictures occur often in this area, for example Landau levels are determined by solving the Schrödinger equation with a harmonic oscillator potential. Thus a simple physical model which emphasizes geometry in the problem is constructed for a Hall system and it is shown that solutions can be found. A wavefunction is obtained under some specific assumptions. It will be seen that some physical properties that are very relevant can be established from the model; in particular, the quantization of the Hall resistance, (1) can be obtained. To begin to set up the model some more physical concepts need to be introduced. Let us proceed to this $[5,6]$.

\section{Setting up the Model-Composite Particles}

Let $\Psi(x)$ be the electron field. An anyon may be thought of as a flux carrying a boson or fermion quantum number. A composite-particle field $\phi(\vec{x})$ is defined by an operator phase transformation

$$
\phi(\vec{x})=e^{-i m \Theta(\vec{x})} \Psi(\vec{x}) .
$$

The phase field $\Theta(\vec{x})$ is defined by

$$
\Theta(\vec{x})=\int d^{2} y \vartheta(\vec{x}-\vec{y}) \rho(\vec{y}),
$$


where $m$ in (3) is an integer and $\vartheta(\vec{x}-\vec{y})$ in (4) is the angle made between the vector $\vec{x}-\vec{y}$ and the $x$-axis; $\rho$ represents anyon density. The effect of the operator phase transformation (3) is to attach $m$ flux quanta to each electron. Composite particles experience the effective magnetic field $\mathrm{B}_{\text {eff }}(\vec{x})$ described by the potential $\mathrm{A}_{j}(\vec{x})$, where $\mathrm{A}_{j}(\vec{x})$ depends on the external vector potential $A_{j}^{\text {ext }}(\vec{x})$ and a field $C_{k}(\vec{x})$, which is an auxiliary field determined solely by the density $\rho(\vec{x})$,

$$
A_{j}(\vec{x})=A_{j}^{e x t}(\vec{x})+C(\vec{x}) .
$$

Therefore, from (5), it follows that

$$
B_{e f f}(\vec{x})=-\epsilon_{i j} \partial_{i} A_{j}(\vec{x})=B_{\perp}-m \phi_{D} \rho(\vec{x}),
$$

and so the effective magnetic flux is the sum of the real magnetic flux and a term which can be regarded as a Chern-Simons flux.

Now suppose that $A_{j}(\vec{x})$ in (4) satisfies the Coulomb gauge condition

$$
\partial_{j} A_{j}(\vec{x})=0 .
$$

It is possible to express $A_{j}(\vec{x})$ in terms of a scalar field $A(\vec{x})$ as

$$
A_{j}(\vec{x})=-\frac{\hbar}{e} \epsilon_{j k} \partial_{k} \mathrm{~A}(\vec{x}) .
$$

This conclusion is only possible in a planar geometry. Substituting (8) into $B_{\text {eff }}(\vec{x})$, the field $\mathrm{A}(\vec{x})$ can be regarded as the scalar potential of the effective magnetic field,

$$
B_{\text {eff }}(\vec{x})=-\frac{\hbar}{e} \nabla^{2} \mathrm{~A}(\vec{x}) .
$$

This is basically the type of constraint we would like to apply in order to solve (2); that is, by taking a particular reasonable form for $B_{\text {eff }}(\vec{x})$.

The state vector $\Psi$ is assumed to fully or very nearly characterize the electronic state of the system. The total free charge is given by

$$
Q=e \int_{s}|\Psi|^{2} d^{2} x .
$$

The steady state time-independent wavefunction is given by

$$
\psi=e^{-i E t / \hbar} \psi_{0},
$$

where $\Psi_{0}$ is time-independent and will have to satisfy the time-independent Schrödinger equation

$$
\mathrm{H} \psi_{0}=E \psi_{0} .
$$

Let us incorporate an additional assumption into the construction of this model here. Let us suppose that we can write $B_{\text {eff }}(\vec{x})=B(\vec{x})$ in the following form

$$
B(\vec{x})=|k \Psi|^{2},
$$

where $k$ is related to the total magnetic flux through the surface; that is, the number of flux quanta of the magnetic field and other constants. The magnetic flux density affects the electronic states as it modifies the Hamiltonian. Of course, the Hamiltonian is modified by the vector potential, which in a simply-connected domain is given by the usual formula $\nabla \times A=B(x)$. For example, suppose we write and use (12) in the form

$$
B(\vec{x})=a\left|\Psi_{0}\right|^{2},
$$

and $a$ is a constant which satisfies

$$
\Phi=\int_{S} B(\vec{x}) d^{2} x=\int_{S} a\left|\Psi_{0}\right| d^{2} x=a \frac{Q}{e}=a N
$$

In (14), $N$ is the number of relevant current carrying charge quanta. Moreover, let $M$ denote the number of magnetic flux quanta, which means the total flux can be written as

$$
\Phi=M \frac{h}{2 e} .
$$

When the flux and charge are quantized, these results imply that $a$ is a fraction which can be expressed in terms of the flux quantum

$$
a=\frac{M}{N} \frac{h}{2 e} .
$$

On a simply connected region, the vector potential can be represented as a one-form given in terms of a single function $\varphi$, which stands for $A$ here, as

$$
A=\varphi_{x} d y-\varphi_{y} d x .
$$

Using (17), the magnetic field can be calculated and then (13) yields a constraint equation

$$
\varphi_{x x}+\varphi_{y y}=a\left|\Phi_{0}\right|^{2} .
$$

\section{Solution of the Schrödinger Equation}

The main objective here is to solve the time-independent Schrödinger equation coupled with Equation (18) to obtain $\Psi$. Of course, vector potential (17) appears in the Schrödinger equation, as can be clearly seen from (2). This procedure will lead to a nonlinear equation; however, it will be found that solutions with the correct physical properties can be determined in closed form. Keeping the first term in (2), the left hand side without the overall multiplicative constant applying (17) leads to

$$
-\left(\partial_{x}^{2}+\partial_{y}^{2}\right) \Psi_{0}+2 \mathrm{i} \frac{e}{\hbar}\left(-\varphi_{y} \Psi_{0, \mathrm{x}}+\varphi_{x} \Psi_{0, \mathrm{y}}\right)+\frac{e^{2}}{\hbar^{2}}\left(\varphi_{x}^{2}+\varphi_{y}^{2}\right) \Psi_{0}
$$


Therefore (11) written out in full takes the form,

$$
\begin{aligned}
& -\hbar^{2}\left\{-\left(\partial_{x}^{2}+\partial_{y}^{2}\right) \Psi_{0}+2 \mathrm{i} \frac{e}{\hbar}\left(-\varphi_{y} \Psi_{0, \mathrm{x}}+\varphi_{x} \Psi_{0, \mathrm{y}}\right)+\frac{e^{2}}{\hbar^{2}}\left(\varphi_{x}^{2}+\varphi_{y}^{2}\right) \Psi_{0}\right\} \\
& =2 m E \Psi_{0} .
\end{aligned}
$$

Now the problem takes the form of finding solutions to (19) subject to the condition (18) This will not be done in a completely general way, but with some assumptions which will lead to a physically relevant result.

Suppose the electron system describes a rectangular geometry in the xy plane. Moreover, let $\Psi_{0}$ have a plane wave dependence in the $\mathrm{x}$ direction, so solutions which have the structure

$$
\Psi_{0}(x, y)=e^{i k x} \theta(y) .
$$

is sought where $\theta(y)$ is a real function of $y$. Let us take the function in the vector potential to be independent of $x$,

$$
\varphi=\varphi(y)
$$

The derivatives of $\Psi_{0}$ can be calculated based on (20) and then substituted into (19),

$$
\begin{aligned}
& -\hbar^{2}\left(-k^{2} \theta(y)+\theta_{\mathrm{yy}}(y)\right)+2 \mathrm{e} \hbar k \varphi(y) \theta(y)+e^{2} \varphi_{y}^{2} \theta(y) \\
& =-2 m E \theta(y) .
\end{aligned}
$$

This takes the form of a second order equation for $\theta(y)$, but it is coupled to $\varphi(y)$ in (17),

$$
-\hbar^{2} \theta_{\text {yy }}(y)+\left(\hbar k+e \varphi_{y}\right) \theta(y)=-2 m E \theta(y) .
$$

If $\varphi$ is assumed to have the form (21), then $\varphi_{x x}=0$ and (18) assumes the simple form

$$
\varphi_{\mathrm{yy}}=a \theta^{2}(y) \text {. }
$$

Since the right-hand side of (24) depends only on $y$, (24) can be integrated once to obtain $\varphi_{\mathrm{y}}$, which appears in (23), in terms of $\theta(y)$ as

$$
\varphi_{\mathrm{y}}(y)=a \int_{y_{0}}^{y} d \tau \theta^{2}(\tau) .
$$

Imposing $\varphi_{y}(0)=y_{0}$. Substituting (25) into (23), this coupled system is reduced to the following nonlinear eigenvalue problem

$$
-\theta_{\mathrm{yy}}(y)+\left(k+\frac{e}{\hbar} a \int_{y_{0}}^{y} d \tau \theta^{2}(\tau)\right)^{2} \theta(y)=-\frac{2 m E}{\hbar^{2}} \theta(y)
$$

Therefore, the dependent variable in (26) is $\theta(y)$. In addition to (26), it is useful to write down a decoupled version which is obtained by introducing a new variable $\sigma(y)$ given by

$$
\sigma(y)=k+\frac{e}{\hbar} a \int_{y_{0}}^{y} d \tau \theta^{2}(\tau) .
$$

Equation (26) can be written in the form of a pair of equations as follows,

$$
\sigma_{\mathrm{y}}(y)=\frac{e}{\hbar} a \theta^{2}(y),-\theta_{\mathrm{yy}}(y)+\sigma^{2}(y) \theta(y)=-\frac{2 m E}{\hbar^{2}} \theta(y) .
$$

The Hall resistance for this two-dimensional system can be calculated based on (28), in fact it can be written in terms of $\sigma(y)$. The geometry is that of a rectangular plate with edges which are parallel to the $x$ and $y$-axes. To be consistent with (20), where the $x$-dependence in $\Phi_{0}$ is assumed to be a plane wave, only the $y$ dimension will be of significance here. The terminations for integration localized at fixed $y$-coordinates, are termed the left (L) and right (R) edges of the geometry. The Hall potential is defined as the difference of potentials between these two edges of the rectangle. In fact, the Hall potential can be obtained from (26), or better in terms of the solution for $\sigma(y)$ by means of

$$
V_{\mathrm{H}}=\frac{\hbar^{2}}{2 m e}\left(\sigma^{2}(R)-\sigma^{2}(L)\right),
$$

where $\mathrm{R}$ and $\mathrm{L}$ refer to right and left. Only the longitudinal $x$ or plane wave component of the current density contributes

$$
j_{\mathrm{x}}=\frac{e}{m} \operatorname{Re} \psi_{0}(i \hbar \vec{\nabla}+e \overrightarrow{\mathrm{A}}) \psi_{0}=-\frac{e \hbar}{m} k+\frac{e}{\hbar} a \int_{y_{0}}^{y} d \tau \theta^{2}(\tau) \theta^{2}(y) .
$$

The potential $V_{\mathrm{H}}$ is transverse to the current. From (28), since $\theta^{2}$ can be related to $\sigma_{\mathrm{y}}$, the current density can be represented entirely in terms of the variable $\sigma$ as

$$
j_{\mathrm{x}}=-\frac{e h}{m} \sigma \theta^{2}=-\frac{\hbar^{2}}{a m} \sigma \sigma_{\mathrm{y}}=-\frac{\hbar^{2}}{2 a m}\left(\sigma^{2}\right)_{y} .
$$

Integrating $j_{\mathrm{x}}$ and using the definition of $V_{\mathrm{H}}$ given in (29), $I_{\mathrm{x}}$ can be related to $V_{\mathrm{H}}$ as follows,

$$
I_{\mathrm{x}}=\int_{R}^{L} j_{\mathrm{x}} d y=-\frac{\hbar^{2}}{2 a m} \int_{R}^{L}\left(\sigma^{2}\right)_{y} d y=\frac{\hbar^{2}}{2 a m}\left(\sigma^{2}(R)-\sigma^{2}(L)\right)=\frac{e}{a} V_{\mathrm{H}} .
$$

By means of (16), the quantity a can be eliminated from (31) to produce the following remarkable formula,

$$
I_{\mathrm{x}}=\frac{N}{M} \frac{2 e^{2}}{h} V_{\mathrm{H}} .
$$

The result in (32) immediately implies the Hall resistance is quantized according to,

$$
R_{\mathrm{H}}=\frac{V_{\mathrm{H}}}{I_{\mathrm{x}}}=\frac{M}{N} \frac{h}{2 e^{2}} \text {. }
$$


Finally, it will be shown that a wavefunction $\psi_{0}$ can be determined based on the coupled system (28). In fact, the coupled equations in (28) can be combined into a single nonlinear differential equation for the function $\sigma(y)$, from which $\theta(y)$ can be determined. To begin to do this, differentiate the first equation in (28) and then divide this by $\sigma_{\mathrm{y}}$ to obtain

$$
\frac{\sigma_{\mathrm{yy}}}{\sigma_{\mathrm{y}}}=2 \frac{\theta_{\mathrm{y}}}{\theta} \text {. }
$$

Differentiating both sides of this, there follows

$$
2 \frac{\theta_{\mathrm{yy}}}{\theta}-2 \frac{\theta_{y}^{2}}{\theta^{2}}=\frac{\sigma_{\mathrm{yyy}}}{\sigma}-\frac{\sigma_{y y}^{2}}{\sigma_{y}^{2}} .
$$

Squaring both sides of (34), an additional expression for $\theta_{y}^{2} / \theta^{2}$ is obtained. Substituting this into the right hand side of (35),

$$
\frac{\theta_{\mathrm{yy}}}{\theta}=\frac{\sigma_{\mathrm{yyy}}}{2 \sigma_{\mathrm{y}}}-\frac{1}{4}\left(\frac{\sigma_{\mathrm{yy}}}{\sigma_{\mathrm{y}}}\right)^{2} .
$$

From the second equation in (28), upon dividing by $\theta$, it follows that

$$
\frac{\theta_{\text {yy }}}{\theta}=\sigma^{2}+\frac{2 \mathrm{mE}}{\hbar^{2}} .
$$

Substituting (37) into (36), a third order nonlinear equation in terms of the independent variable $\sigma$ results,

$$
\frac{\theta_{\text {yyy }}}{\theta_{y}}=\frac{1}{2}\left(\frac{\sigma_{\text {yy }}}{\sigma_{y}}\right)^{2}+2 \sigma^{2}-2 \hat{E},
$$

where we put

$$
\hat{E}=-\frac{2 \mathrm{mE}}{\hbar^{2}}
$$

A general solution to (38) may not be possible, however, something can be done. Note that upon omitting $\left(2 \sigma^{2}-2 E\right)$ from (38), the equation can be integrated. Thus, we have $\left(\ln \left(\sigma_{\mathrm{yy}}\right)\right)_{y}-\frac{1}{2}\left(\ln \left(\sigma_{\mathrm{y}}\right)\right)_{y}=0$, and integrating gives $\left(\sigma_{\mathrm{yy}}\right)^{2}-\tilde{c} \sigma_{\mathrm{y}}=0$. This can be integrated as well to give $\sigma(\mathrm{y})=\frac{1}{3 c_{1}}\left(c_{1} \mathrm{y}+c_{2}\right)^{3}+c_{3}$. A specific physically realistic solution to the general form of (38) can be approached as follows. The first equation in (28) implies that the sign of $\sigma(\mathrm{y})$ is determined by $a$, therefore, when $\theta$ does not vanish, $\sigma$ must be a monotonic function. Consequently, one way in which a class of solution can be obtained is to consider the case in which $\sigma_{\mathrm{y}}$ is only a function of $\sigma$,

$$
\sigma_{\mathrm{y}}=w(\sigma)
$$

In fact, $g(\sigma)$ can be determined explicitly. Differentiating both sides of (40) with respect to $y$, we get

$$
\sigma_{\text {yy }}=\sigma_{\mathrm{y}} w_{\sigma}=\mathrm{w} w_{\sigma}, \sigma_{\text {yyy }}=w\left(w_{\sigma}^{2}+\mathrm{w}_{\sigma \sigma}\right) \text {. }
$$

Substituting (40) and (41) into (38) gives rise to the following equation for $w$,

$$
\mathrm{w}_{\sigma \sigma}+\frac{1}{2} w_{\sigma}^{2}=2\left(\sigma^{2}-\hat{E}\right),
$$

Clearly (42) is nonlinear, however, there is a way to produce a solution which is physically reasonable. There exists a quadratic polynomial solution for $\mathrm{w}$ which can be expressed in terms of $\sigma$ as

$$
\mathrm{w}(\sigma)=\alpha \sigma^{2}+\beta \sigma+\gamma
$$

These constants can be specified upon substitution in (42), and it will constitute a solution provided that $\beta=0$ and

$$
\mathrm{w}(\sigma)= \pm \frac{1}{\sqrt{2}} \sigma^{2}-\sqrt{2} \hat{E} .
$$

Taking (43) and replacing the result in (40), it is clear the resulting equation can be separated to give

$$
\int \frac{\sqrt{2} d \sigma}{ \pm \sigma^{2}-2 \hat{E}}=\mathrm{y}+\mathrm{c} .
$$

The negative sign gives a tangent function solution which will be prone to have poles and can be written

$$
\sigma(y)=-\sqrt{2 \hat{E}} \tan (\sqrt{2}(\mathrm{y}+\mathrm{c})) .
$$

However, the other choice of sign in (44) gives rise to the result,

$$
-\frac{1}{\sqrt{\hat{E}}} \arctan \left(\frac{\sigma}{\sqrt{2 \hat{E}}}\right)=\mathrm{y}+\mathrm{c} .
$$

This can be solved explicitly for the function $\sigma(y)$,

$$
\sigma(y)=-\sqrt{2 \hat{E}} \tanh (\sqrt{\hat{E}}(\mathrm{y}+\mathrm{c}))=-\sqrt{2 \hat{E}} \frac{1-\mathrm{Ce}^{-2 \sqrt{\hat{E} \mathrm{y}}}}{1+\mathrm{Ce}^{-2 \sqrt{\hat{E} \mathrm{y}}}} .
$$

By differentiating (45), an expression for $\theta^{2}(y)$ is obtained. The function $\theta(y)$ which we need to write the wavefunction (20) is found from the square root of this, namely

$$
\theta(y)= \pm 2 \cdot 2^{\frac{1}{4}}\left(\mathrm{C}_{1} \frac{\hbar \hat{E}}{e a}\right)^{\frac{1}{2}} \frac{e^{-\sqrt{\hat{E} \mathrm{y}}}}{1-\mathrm{C}_{1} e^{-2 \sqrt{E \mathrm{y}}}}
$$

The wavefunction is then determined using (46) by means of, 


$$
\Psi=e^{-i E t / \hbar} e^{i k x} \theta(y) .
$$

This is a bounded function on any right half axis and square integrable over the rectangular area. Thus there exists a solution with the desired physical properties.

Therefore, it has been seen how (1) emerges and that physical classes of solutions to (2) can be investigated. Most importantly, a link between the wavefunctions implied by the model and the calculation of a corresponding resistence for the model has been shown.

\section{Conclusions}

An elementary model for the quantum Hall effect has been developed. It is known in this field that simple models based on Schrödinger equations can be very useful in studying the effect. For example, the equation is solved with the harmonic oscillator potential to describe and obtain the energies of Landau levels. The model emphasizes several aspects of the geometry of the system in obtaining the results $(32,33)$. It is quite interesting that a single particle Schrödinger equation can be obtained and solved in closed form, and which incorporates a sig- nificant amount of the physics involved.

\section{References}

[1] J. K. Jain, "Composite Fermions, Perspectives in Quantum Hall Effects," In: S. D. Sarma and A. Pinczuk Eds., J. Wiley and Sons, New York, 1987.

[2] K. von Klitzing, G. Dorda and M. Pepper, "New Method for High-Accuracy Determination of the Fine Structure Constant Based on Quantized Hall Resistance," Physics Review Letters, Vol. 45, No. 6, 1980, pp. 494-497.

[3] R. B. Laughlin, "Quantized Hall conductivity in Two dimensions," Physical Review B, Vol. 23, No. 10, 1981, pp. 5632-5633.

[4] R. E. Prange and S. M. Girvin, "The Quantum Hall Effect," Springer Verlag, New York, 1987.

[5] Z. F. Ezawa, "The Quantum Hall Effects, Field Theoretical Approach and Related Topics," World Scientific, Singapore, 2000.

[6] R. B. Laughlin, "Fractional Quantization," Reviews of Modern Physics, Vol. 71, No. 4, 1999, 863-874. 